Bericht über die zur Preisbewerbung eingesandte Arbeit: „Recherches expérimentales sur la valeur de l'équivalent mécanique de la chaleur

\author{
von Hrn. G. A. Hirn in Logelbach bei Colmar".
}

Die Abhandlung zerfällt in zwei wesentlich verschiedene Theile. Der erste enthält die experimentellen Untersuchungen und ihre Discussion, der zweite, welcher "Conclusions générales” überschrieben ist, enthält allgemeine philosophische Betrachtungen, welche zwar die Resultate des experimentellen Theiles als Ausgangspunkt genommen haben und mit ihnen zusammenhängen, aber doch aufserhalb der von der Gesellschaft gestellten Aufgabe liegen. Der Verfasser sagt selbst am Anfange dieses Abschnitts: „Par sa nature même cette dernière partie de mon travail ne peut aucunement figurer parmi les pièces soumises au concours". Ich glaube daher mich auch in der Beurtheilung auf den erslen Theil, welcher eigentlich für den Concurs bestimmt ist, beschrän. ken zu können.

Der experimentelle Theil besteht aus vier Versuchsreihen, von denen die beiden ersten so nahe zusammenhängen, dafs es zweckmäfsig sein wird, sie auch bei der Beurtheilung zu vereinigen.

Erste Versuchsreihe.

Wärueerzeugung durch Reibung.

Eine vollkommen cylindrische, äufserlich glatt polirte Trommel von Gufseisen, welche um ihre horizontal liegende Axe drehbar ist, ist auf dem oberen Theile ihrer Cylinderfläche von einem Metallkörper bedeckt, dessen untere Fläche die Hälfte eines Hohlcylinders bildet, und sattelartig auf der Trommel aufliegt, so dafs, 
wenn die Tronmel gedreht wird, zwischen ihr und dem ruhenden Saltel eine Reibung stattindet. Zwischen den sich reibenden Flächen befand sich bei diesen Versuchen ein Schmiermittel, wozu nach einander verschiedene Fettarten angewandt wurden.

Die zur Ueberwindung der Reibung erforderliche Kraft wurde ganz ähnlich wie bei einem Prony'schen Zaume beslimmt, nämlich durch das Gewicht, welches man an dem Endpunkte eines Hebelarmes anbringen mufste, um zu bewirken, dafs der Sattel bei der Drehung der Trommel nicht mit gedreht wurde.

Um die erzeugte Wärme zu bestimmen, wurde die Trommel zum Theil mit Wasser gefüllt, weiches entweder während des Drehens durch gleichförmiges Zufliefsen von kaltem Wasser und entsprechendes Abfliefsen des erwärnten Wassers fortwährend erneuert wurde, oder unverändert in der Trommel blieb, bis es durch die Reibung eine constante Temperatur angenommen hatte, oder endlich bei hoher Temperatur in die Trommel gebracht wurde, und sich während des Drehens abkühlte, indem es sich derselben constanten Temperatur näherte.

Jede dieser drei Beobachtungsweisen wurde mehrmals wiederholt, so dafs sie im Ganzen 32 Werthe für das Arbeilsäquivalent der Wärme lieferten, welche alle gut mit einander übereinstinmen, indem sämmtliche Zahlen zwischen 359 und 382 liegen. Das Mittel ist als Arbeit für eine Wärmeeinheit in Kilogrammmetern ausgedrückt 371,6.

\section{Zweite Versuchsreihe.}

Wărmeerzeugung bei der Trennung der Körpertheile.

Stücke von verschiedenen Metallen, welche zuvor jedes mit einer cylindrischen Höhlung versehen waren, wurden, während sie sich in einem Wassercalorimeter befanden, weiter ausgebohrt. Die Kraft, welche zum Bohren verwandt werden mufste, wurde dadurch bestimmt, dafs, während der Bohrer um eine senkrechte Axe gedreht wurde, das Metallstück ebenfalls um eine senkrechte Axe, welche die Fortsetzung der Axe des Bohrers bildete, drehbar war, und mit einem horizontalen Arme versehen war, an dessen Endpunkte eine horizontal fortlaufende und dann über eine 
Rolle gehende Schnur befestigt war, welche ein Gewicht trug. Dieses Gewicht hinderte die Drehung des Metallstücks, und die Kraft, mit welcher der Bohrer dasselbe zu drehen suchte, konnte also dadurch gemessen werden.

Die einzelnen Resultate dieser Versuchsreihe führt der Verfasser nicht an, sondern nur den Mittelwerth, welcher ist: 425.

Dieser durch die zweite Versuchsreihe gewonnene Werth stimmt mit dem durch die erste Versuchsreihe gewonnenen nicht überein. Als einen Grund dafür lührt der Verfasser an, dals beim Bohren ein intensiver Ton hervorgebracht wurde, welcher bei der Reibung nicht eintrat. Da indessen dieser Grund doch nicht hinreicht, um die gefundenen Unterschiede vollständig zu erklären, so neigt sich der Verfasser zu der Ansicht, dafs der Unterschied in der Natur der Sache selbst läge, indem das Arbeitsäquivalent der Wärme nicht unter allen Umständen gleich sei, sondern von der Art des Processes abhänge.

Ich glaube jedoch, dafs man, ohne die Correctheit der Beobachtungen in Zweifel zu ziehen, doch diesen Schlufs nicht als nothwendig zuzugeben braucht, indem bei den Versuchen noch andere von dem Verfasser nicht berücksichtigte Uınstände vorkommen, welche möglicher Weise die gefundene Differenz erklären können.

Zunächst ist nicht berücksichtigt, dafs bei der ersten Versuchsreihe das in der Trommel befindliche Wasser bei der Drehung fortwährend umgerührt wurde und diese Reibung des Wassers selbst ebenfalls etwas dazu beitragen mufste, seine Temperatur zu erhöhen. Diese Wärmemenge aber, welche an sich schon unbedeutend ist, konnte nur bei der dritten Beobachtungsweise dieser Reihe zur vollständigen Wirksamkeit kommen, und mufste hier dazu beitragen, den Werth des Arbeitsäquivalentes etwas zu klein zu machen. Bei den beiden ersten Beobachtungsweisen ist das Resultat der letzten dazu benutzt, um die Wärmeverluste des Apparates nach aufsen zu bestimmen. Dadurch ist dieselbe Wärmemenge noch einmal im entgegengesetzten Sinne in die fiormeln gekommen, und mufs sich daher zum Theil selbst aufgehoben haben.

Fortschr. d. Pliys. XI. 
Aufserdem ist aber noch ein anderer wesentlicher Umstand zu erwähnen.

Bei der Bestimmung des Arbeitsäquivalentes der Wärme ist es eine Hauptbedingung, dals die Körper, welche zum Versuche angewandt werden, dabei ihren Zusland nicht ändern, oder falls eiñe Zustandsänderung stattgefunden hat, die dazu verwandte Arbeit genau in licchuung gebracht wird. Diese Bedingung ist aber bei den vorliegenden Versuchen nicht vollständig erfüllt.

Bei der zweiten Versuchsreihe ist es offenbar, dafs zur Trennung der ausgebohrten Metalitheilchen von der übrigen Masse ein gewisser Arbeitsaufwand gehörte, und dafs also nicht die ganze Arbeit derjenigen Kraft, welche den Bohrer drehte, zur Wärmeerzeugung verwandt wurde. Die mit der Wärme verglichene Arbeit ist also zu grofs genommen, und demgemäls mufs auch die gefundene Zahl 425 als etwas 24 grofs betrachtet werden.

Bei der ersten Versuchsreihe haben sich zwar die Maschinentheile selbst nicht wesentlich geändert, aber, wie schon erwähnt, war die Reibung der Metallfächen keine unmittelbare, sondern es befand sich ein Schıniermittel zwischen ihnen, und dieses hat sich jedenfalls während der Reibung in seinem Zustande geändert. Welcher Art diese Aenderung auch sein mag, so können jedenfalls Versuche, bei welchen der Körper, an welchem die Hauptreibung slattfand, eine Zustandsänderung erlitt, welche bei der Berechnung nicht mit berücksichtigt ist, keinen Anspruch auf so strenge Gültigkeit ihrer Resultate machen, un als Einwurf gegen dos allgemeine Princip von der Aequivalenz von Wärme und Arbeit zu dienen.

Ich will, ohne ein Gewicht auf die Richtigkeit dieser Erklärung zu legen, einen möglichen Fall als Beispiel anführen. Nimmt man an, es werde in dem Fette theils durch die Wärme, theils durch die Berührung mit den Metallflächen eine chemische Veränderung eingeleitet, welche während der Reibung langsam vor sich gehe, und bei welcher Wärme erzeugt würde, so würde von der ganzen gemessenen Wärme ei) Theil nicht unmittelbar von der gemessenen Arbeit erzeugt sein, und wenn man diesen Theil der Wärme vor der. Vergleichung in Abzug gebracht hätte, no 
würde der Werth des Arbeitsäquivalentes der Wärme gröfser als 371,6 ausgefallen sein.

Für eine ähnliche Wirkung des Fettes sprechen auch einige weiterhin vom Verfasser selbst angeführte Erscheinungen.

Er hat mit dem Apparate der ersten Versuchsreihe auch Versuche angestelit, bei welchen kein Felt angewandt wurde, sondern die Metallfächen selbst sich rieben. In diesen Fällen war die Bestimmung der Arbeit weniger genau möglich, weil die Reibung zu ungleichmälsig war; indessen glaubt der Verfasser sich überzeugt zu haben, dafs diese Versuche für das Arbeitsäquivalent einen Werth geben, welcher bedeulend gröfser ist als 371,6 und sich den Zahlen 410 bis 420 nähert.

Ebenso hat er gefunden, dafs, wenn man frisches Fett zwischen die Metallfächen brachte, dieses nicht gleich die Reibung verminderte, sondern Anfangs sogar vermehrte, und erst allmälig in den Zustand kam, in welchem es die geringste Reibung gab, die dann für längere Zeit nahe constant blieb. Benutzte man die Zeit, bevor dieser Zustand erreicht war, zur Bestimmung des Aequivalentes der Wärme, so erhielt man ebenfalls Zahlen, welche über 371,6 lagen, und sich einer Zahl näherten, die wenigstens 400 war.

Alle diese Gründe scheinen zu dem Schlusse zu berechtigen, dafs der Unterschied zwischen den durch die erste und zweite Versuchsreihe gefundenen Zahlen nur auf Nebenumständen beruht, und dafs man als Resultat dieser Versuche eine Zahl annehmen kann, welche $z$ wischen jenen beiden liegt, wahrscheinlich etwas über 400. Dieses Resultat stimunt mit dem von Joule durch Reibung gefundenen Werthe 423,55 hinlänglich überein.

\section{Dritte Versuchsreihe. \\ Wärmeverbrauch in Dampfmaschinen.}

In dieser Versuchsreihe, welche meiner Ansicht nach die wichtigste ist, wurde bestimmt, wie viel Wärme dem Dampfe mitgetheilt werden mufste, um inn in den Zustand zu bringen, in welchem er in den Cylinder trat, und wie viel Wärme der Danpf 
XX Nachrichten über die physikalische Gesellschaft.

nach dem Austritt aus dem Cylinder im Condensator wieder abgab, und die gefundene Differenz wurde mit der gethanen Arbeit verglichen.

Versuche dieser Art sind aufserordentlich schwierig, zumal wenn sie, wie der Verfasser es gethan hat, an Maschinen von über hundert Pferdekräften angestellt werden. Der Verfasser sagt ganz richtig, dafs Beobachtungen an einer solchen Maschine sehr verschieden sind von denen an einem physikalischen Apparate, bei welchem der Experimenlator die Erscheinungen leicht zur gewünschten Zeit und mit beliebigen Modificationen hervorbringen kann, und dafs sie mehr den Beobachtungen einer $\mathrm{Na-}$ turerscheinung gleichen, die man in der Weise, wie sie einmal stattfindet, betrachten, und die Einzelnheiten während ihres Verlaufes erfassén mufs.

Der Verfasser hat diese Schwierigkeiten durch geschickte und zweckmälsige Anordnung seiner Versuche, und durch Sorgfalt in ihrer Ausführung zum grofsen Theile zu überwinden gewufst, wobei er sich nicht auf eine bestimmte Art des Ganges der Maschine beschränkte, sondern den Gang mannigfach variiren liefs.

Er stellte seine Untersuchungen mit zwei Maschinen an, deren eine eine gewöhnliche Maschine mit einem Cylinder war, die andere eine Woorf'sche Maschine mit zwei Cylindern, bei welcher der Dampf, nachdem er in dem einen Cylinder auf dẹn Stempel seinen vollen Druck ausgeübt hat, in den zweiten gröfsern Cylinder tritt, und hier während der Expansion wieder auf den Stempel wirkt. Beide Maschinen wurden entweder so angewandt, dafs der Dampf im gesättigten Zustande aus dem Kessel in den Cylinder trat, oder dafs er vorher noch bis etwa $250^{\circ}$ überhitzt wurde. Ferner waren bei der Woolf'schen Maschine die beiden Cylinder von einem weiteren Mantel umgeben, so dafs der Zwischenraum mit Dampf gefüllt sein konnte, um die Cylinder auf bestimmter Temperatur zu erhalten. Dieser Zwischenraum wurde bei den Versuchen entweder mit überhitztem Dampfe oder mit gesättigtem Dampfe oder nur mit Luft gefüllt erhalten.

Es würde zu weit führen, auf die Details der Versuche hièr einzugehen, und ich will nur erwähnen, dafs sie viele interessante 
und für die Kenntnifs der Vorgänge in einer Dampfmaschine wichtige Resultate geliefert haben. Als ein Hauptergebnifs für die gestellte Aufgabe ist zunächst anzuführen, dafs der Umstand, dafs der Dampf nach seiner Arbeit im Condensator weniger Wärme abgiebt, als er im Kessel empfangen hat, auch wenn inan alle Wärmeverluste nach aufsen schon in Abzug gebracht hat, ein Umstand, der zwar theoretisch nothwendig war, aber experimentell, so viel ich weifs, noch nirgends nachgewiesen ist, durch diese Versuche vollkommen festgestellt ist.

Die Arbeit, welche von den Maschinen während der Versuche gethan wurde, ist sowohl mittelst des Prony'schen Zaums, als

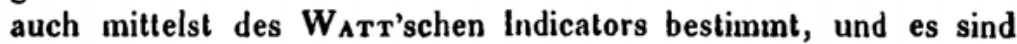
besondere Versuche darüber angestellt, um festzustellen, wie sich die Angabe dieser Instrumente zu der ganzen vom Dampfe im Cylinder gethanen und durch Reibung noch nicht verminderten Arbeit verhält.

Indem nun der Verfasser die gewonnene Arbeit mil der verbrauchten Wärme verglichen hat, ist er merkwürdiger Weise in einen offenbaren Irrthum verfallen. Er nimmt nämlich an, dafs nur zu dem Theile der Arbeit, welche der Dampf während seiner Expansion thut, Wärme verbraucht werde, und berechnet daher das Arbeitsäquivalent der Wärme in der Weise, dafs er nicht die ganze gewonnene Arbeit, sondern nur den während der Expansion gethanen Theil der Arbeit durch die verbrauchte Wärme dividirt. Dadurch mufsten natürlich die Zahlen viel zu klein werden und auch bei den verschiedenen Versuchen sehr verschieden ausfallen, indem der von der Expansion herrührende Theil der Arbeit nicht bei allen Versuchen in demselben Verhältnisse zur ganzen Arbeit stand.

Dafs diese Ansicht der mechanischen Wärmetheorie vollständig widerspricht, ergiebt sich am einfachsten daraus, dafs man hiernach bei einer Maschine, welche ohne Expansion arbeitet, Arbeit ohne Wärmeverbrauch erhalten würde.

Es läfst sich aber auch leicht nachweisen, auf welche Weise dieser Irrthum bei dem Verfasser entstanden ist. Er sagt nämlich zur Rechtfertigung jener Annahme: wenn Dampf sich bei demselben Drucke niederschlägt, bei welchem er entstanden ist, 
so giebt er beim Niederschlag eben so viel Wärme ab, als ihm bei seiner Entstehung mitgetheilt werden unufste. Dieser Satz ist allerdings richtig, findet aber auf die Danpfmaschine keine Anwendung.

Wenn bei einer Dampfmaschine, welche ohne Expansion arbeitet, der Dampf den Cylinder an der einen Seite des Stempels ganz angefüllt hat, und nun diese Seite mit dem Condensator in Verbindung geselzt wird, so strömt nur der erste Theil des Dampfes mit seinem vollen Drucke in den Condensalor, und der folgende mit allmälig abnehmendem Drucke, und auch dieser Druck entsteht nur dadurch, dafs der noch im Cylinder befindliche Dampf sich ausdehnt, und bei dieser Ausdehnung mufs der Dampf schon im Cylinder sich bedeutend abkühlen, und sogar, wenn er nicht überhitzt ist, oder ihm von aufsen Wärme zugeführl wird, sich schon im Cylinder zum Theil niederschlagen. Um die in jenem Satze enthaltene Bedingung zu erfüllen, mürste der Stempel während des Ausströmens mit solcher Geschwindigkeit zurückgehen, dafs der noch in Cylinder befindliche Dampf immer auf dem vollen Drucke erhalten würde. Dann würde aber auch die Gegenkraft, welche der Stempel auf dem Rückgange zu überwinden hälte, eben so grofs sein als die treibende Kraft auf dem Hingange und nichts an Arbeit gewonnen sein. Hätte der Verfasser seine Versuche auch auf eine Maschine ohne Expansion ausgedehnt, so würde er ohne Zweifel auch bei dieser gefunden haben, dafs die abgegebene Wärmemenge geringer ist als die aufgenommene.

Ich habe versucht, diesen Fehler aus den Resultaten zu eliminiren, was freilich nur auf unvollkommene Weise möglich war.

Der Verfasser hat nämlich, obwohl sein Augenmerk hauptsächlich auf den 'Theil der Arbeit gerichtet war, welcher während der Expansion gethan wurde, beiläufig auch die ganze Arbeit, welche die Maschine bei jeder Versuchsreihe that, in Pferdekräften angegeben. Diese Zahlen stellen aber die wirklich nutzbare Arbeit dar, wie sie durch den Prony'schen Zaum gegeben wird, in welcher die Reibungen innerhalb der Maschine schon in Abzug gebracht sind. Bei der Rechnung muls aber die volle Arbeit, welche der Dampf im Cylinder thut, bekannt sein. Der Verfar- 
ser hat nun bei der Maschine mit einem Cylinder Versuche angestellt, bei welchen er die volle Arbeit berechnen konnte, und hat diese dann mit der gleichzeitig durch den Prony'schen Zaum angegebenen nutzbaren Arbeit verglichen. Dabei hat er gefunden, dafs die letztere 70 bis 75 Procent der ersteren betrug. Ferner hat er bei der WooLf'schen Maschine Versuche ähnlicher Art mit dem $W_{\Delta T T}$ 'schen Indicator angestellt, von welchem Instrumente er schon früher gesagt hat, dafs es zufällig verhältnifsmäfsig eine eben so grofse Reibung halte wie die untersuchte Dampfmaschine, so dafs die Angaben des Indicators denen des Zaumes gleich zu setzen sind. Auch bei diesen Versuchen mit der Wool.'s'schen Maschine ergab sich, dafs die nutzbare Arbeit 70 bis 75 Procent der ganzen Arbeit betrug.

lch habe daher aus den vom Verfasser fiir die nutzbare Arbeit gegehenen $Z$ ahlen die ganze Arbeil zweimal berechnet, indem sch jene Zahlen einmal mit $\frac{100}{70}$ und das andere Mal mit $\frac{100}{75}$ multiplicirt habe. Mit den auf diese Weise abgeleiteten Arbeitsgröfsen habe ich dann dieselbe Rechnung angestellt wie der Verfasser mit den bei der Expansion gethanen Arbeitsgrölsen. Die Resultate dieser Rechnung sind in der folgenden kleinen Tabelle mil den vom Verfasser berechneten Zahlen zusammengestellt.

Arbeitsäquivalent der Wärme

nach dem Verfasser

berechnet aus der ganzen Arbeit unter Anwendung des Bruches

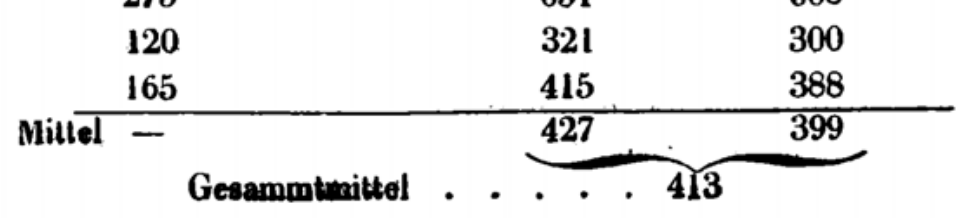


Man sieht, dafs dieses letzte Mittel mit den durch Reibung gefundenen Zahlen, wie sie Joure angiebt und wie sie sich aus den Versuchen des Verfassers selbst als wahrscheinlichstes Resultat ergeben haben, sehr gut übereinstimmt.

Unter den einzelnen Werthen weichen allerdings einige bedeutend von dem Mittelwerthe ab. Es kann sein, dafs dieses zum Theil daraul beruhl, dafs bei der von mir ausgeführten Rechnung Zahlen angewandt werden mufsten, welche der Verfasser nur beiläufig angeführt hat, und welche daher vielleicht weniger genau bestimmt sind als diejenigen, welche der Verfasser zu seinen eigenen Rechnungen bedurfte. Vielleicht würde der Verfasser, wenn er selbst mit Anwendung seines vollständigen Beobachtungsmaterials eine ähnliche Rechnung anstellte wie ich, noch einige Fehler eliminiren, und dadurch die Zahlen in bessere Uebereinstimmung bringen können. Indessen auch so wie sie sind, scheinen mir die Zahlen von grofsem Werthe zu sein. Wenn man die Schwierigkeit von Versuchen dieser Art und die Menge von Nebenumständen, welche dabei mitwirken und nicht immer genau controllirt werden können, berücksichtigt, und ferner bedenkt, dafs die einzelnen Zahlen nicht durch dasselbe Verfahren gewonnen sind, sondern dafs die $9 \mathrm{Zahlen}$ aus 6 verschiedenen Beobachtungsweisen hervorgegangen sind, indem zwei Maschinen, welche in ihrer Construction wesentlich von einander abweichen, angewandt wurden, und jede Maschine wieder unter sehr verschiedenen Umständen arbeitete, so dafs fast bei jeder folgenden Bestimmung andere Fehlerquellen obwalteten als bei der vorigen, so werden diese Abweichungen der einzelnen Zahlen vom Mittel weniger auffällig erscheinen.

Das Gesammtresultat ist meiner Ansicht nach als eine schöne Bestätigung der von Jouke ausgeführten Untersuchungen zu betrachten, welche zugleich eine wesentliche Vervollständigung der bisherigen Versuche bildet, indem diese Bestimmung des Arbeitsäquivalentes die erste ist, welche experimentell aus einem solchen Processe abgeleitet ist, bei dem nicht Wärme durch Arbeit, sondern Arbeit durch Wärme hervorgebracht wird und der diese Wirkung vermittelnde Körper sich schliefslich wieder in seinem Anfangszustande befindet. Aufserdem gewinnt das Re- 
sultat noch dadurch an Interesse, dafs es gerade aus Beobachtungen an der Maschine getwonnen ist, welche in der Praxis am meisten zur Erzeugung von Arbeit durch Wärme angewandt wird, und welche daher einen besonders wichtigen Gegenstand für die Anwendung der mechanischen 'Wärmetheorie bildet, wie sie ja auch als Ausgangspunkt für die Carnot'sche Theorie gedient hat.

\section{Vierte Versuchsreihe.}

\section{Wärmeverbrauch in menschlichen Körper.}

In dieser Versuchsreihe hat der Verfasser theils an sich selbst, theils an anderen Personen untersucht, wie sich die Menge der durch Respiration gebildeten Kohlensäure und die Menge der nach aufsen abgegebenen $W$ ärme ändern, wenn der Körper, statt sich in Ruhe zu befinden, eine gewisse gleichmäfsige Arbeit thut. Es zeigte sich, dafs beide Mengen während der Arbeit grölser waren als während der Ruhe; aber die Zunahme fand nicht in gleichem Verhältnisse statt. Die Wärmeabgabe wuchs in geringerem Verhältnisse als die Kohlensäurebildung, und es mufste also ein Theil der durch den chemischen Process erzeugten Wärme anderweitig verbraucht sein. Indem nun der Verfasser annahm, dafs die fehlende Wärme das Aequivalent der gewonnenen Arbeit sei, bestimmte er daraus das Arbeitsäquivalent der Wärme.

Wenn diese Versuche an sich betrachtet auch sehr interessant sind, und gewils manche für die. Physiologie nützliche Nebenresultate geliefert haben, so scheinen sie mir doch für die Bestimmung des Arbeitsäquivalentes der Wärme von keinem wesentlichen Nutzen zu sein.

Der thierische Körper hat einige Eigenschaften, welche sich einer genauen Bestimmung dieser Art entgegenstellen, unter denen ich nur eine anführen will. Eine Hauptbedingung für eine Maschine, welche zu solchen Versuchen dienen soll, ist die, dafs in der Maschine entweder keine bleibenden Veränderungen stattfinden, oder doch nur Veränderungen, die genau genug bekannt sind, um sie in Rechnung bringen zu können. So bestehen z. B. in einer Dampfmaschine die Veränderungen nur in periodischen 
Zustandsänderungen des Wassers, während die Maschine selbst ungeändert bleibt. Wenn man daher der Maschine immer so viel Wärme im Kessel zuführt, und so viel Wärme in Condensator entzieht, wie zu diesen Veränderungen des Wassers nöthig ist, so kann man sie beliebig lange arbeiten lassen. Das ist aber bein thierischen Körper nicht der Fall. Nach einer gewissen Zeit der Arbeit ist er erschëpft und bedarf der Ruhe, und es würde bis jetzt schwer sein, die Veränderungen, welche in ihm vorgegangen sind und den Zustand der Ersclıöpfung hervorgebracht haben, genau zu bestimmen.

Ferner scheint es mir, als ob in den Versuchen des Verfassers eine bedeutende Fehlerquelle vorhanden ist. $\mathrm{Er}$ hat in Bezug auf den Wärmeverlust nur berücksichtigt, dals der Körper Wärme an seine Uingebung abgiebl (theils durch Strahlung, theils durch Erwärmung der umgebenden Luft), und dafs die ausgeathmete Luft wärmer ist als die eingeathmele, und mehr Wasserdampf enthält, zu dessen Bildung Wärme verbraucht werden mufste. Aufserdem verdunstet der Körper aber auch an seiner ganzen Oberfläche, und dazu wird ebenfalls Wärme verbraucht, und diese Verdunstung ist nicht immer gleich stark, sondern von dem Grade der Feuchtigkeit der Haut abhängig. Da nun wohl anzunehmen ist, dafs die Personen, mit welchen die Versuche angestellt wurden, wenn sie in einem engen Raume, bei einer eigenthümlichen Art der Athmung durch Schläuche, noch eine anstrengende und ihnen ungewohnte Arbeit thun mulsten, dabei mehr oder weniger in Schweifs geriethen, oder wenn auch kein vollständiges Schwitzen eingetreten sein sollte, doch die Haut feuchter geworden ist als während der Ruhe, so kann man daraus schlielsen, dafs auch der durch die Verdunstung verursachte Wärmeverlust während der Arbeit gröfser gewesen ist. Dieser Umstand ist bei der Berechnung vernachlässigt, und es ist also die zu der vermehrten Verdunstung verbrauchte Wärme als fehlend betrachtet und mit zu derjenigen gerechnet, welche zur Arbeit verbraucht ist. Dadurch wurde die Wärmemenge im Verhältnifs zur Arbeit zu grofs, und das Arbeitsäquivalent der Wärme mufste daher zu klein ausfallen.

Es ist möglich, dafs dieser zuletzt erwähnte Umstand allein 
sehon hinreicht, um die bedeutende Abweichung der aus diesen Versuchen für das Arbeitsäquivakent gefundenen Zahlen von den früheren zu erklären. Jedenfalls aber kann diesen Abweichungen kein solches Gewicht beigelegt werden, um irgend einen Schlufs über die Wärmetheorie daraus zu ziehen.

An den bisher besprochenen experimentellen Theil der Abhandlung schliefst sich, wie schon erwähnt, ein anderer mehr philosophischer Theil an, zu welchem der Verfasser dadurch veranlafst ist, dafs er bei der Arbeit der Dampfmaschine und des menschlichen Körpers andere Werthe des Arbeitsäquivalentes der Wärme gefunden zu haben glaubt als bei der Wärmeerzeugung durch Reibung. Mit den Betrachtungen dieses Theiles kann ich mich nicht überall einverstanden erklären. Da aber der Verfasser selbst diesen Theil nicht für den Concurs bestimmt, sondern nur des Zusammenhanges wegen mitgesandt hat, so glaube ich, dafs derselbe bei der Beurtheilung der Abhandlung in Bezug auf den Preis ganz unberiicksichligt bleiben kann.

Als Ergebnifs der vorstehenden Beurtheilung erlaube ich mir bei der Commission folgenden Antrag zu stellen:

Da mehrere der Hauptresultate, zu welchen der Verfasser gelangt ist, nicht als richtig anerkannt werden können, und die Abweichungen zum Theil nicht auf zufälligen Uınständen, sondern auf einer unrichtigen Auffassung des Gegenstandes beruhen, sieht sich die Commission nicht veranlafst, die Arbeit zu krönen. Indem sie jedoch anerkennt, dafs die Untersuchungen im Einzelnen viele Ergebnisse geliefert haben, welche selbst in den Fällen, wo der Verfasser nicht das richtige Endresultat daraus gezogen hat, doch zur Lösung der gestellten Aufgabe wesentlich beitragen und werthvolle Bereicherungen der Wissenschaft bilden, und welche nur durch grofse Geschicklichkeit und Sorgfalt in der Ausführung der Versuche und durch einen bedeutenden Aufwand von Zeit und Kosten gewonnen werden konnten, beschliefst die Commission, dem Verfasser die für den Preis ausgesetzte Summe von 250 Thalern Gold zu überantworten, wobei sie zugleich den Wunsch ausspricht, dafs der Verfasser den Gegenstand noch einmal 
XXvıI Nachrichten über die physikalische Gesellschaft.

aufnehmen möge, um die Endresultate aus seinem Beobachtungsmaterial nach den richtigen Principien der mechanischen Wärmetheorie zu berechnen, und, wo er es für nöthig hält, das Beobachtungsmaterial durch neue Versuche zu vervollständigen, was ihm bei den einmal gemachten Vorarbeiten leichler werden murs als anderen Beobachtern.

Zürich, den 22. März 1857.

\section{R. Clausius.}

